\title{
L'assurance-vie
}

\section{L'alternative sûre!}

Dans un contexte où tout le système financier est au bord du gouffre, la question se pose de savoir où et comment l'on veut placer son argent. Dans l'or? Mais que se passera-t-il avec le cours de l'or après la crise? Sur un compte épargne? Ma foi, il est vrai qu'ils sont finalement garantis par l'Etat dans le cadre de la protection des placements. Dans les obligations? Mais dans quelle mesure la société émettrice est-elle sûre? Et quand est-il finalement de la solution classique fournie par l'assurance-vie? Quel niveau de sécurité représente cette solution de placement?

Toutes les sociétés suisses d'assurance-vie ont l'obligation d'introduire les prétentions financières des contrats d'assurances-vie qu'elles ont conclues dans un fonds de garantie. Ce fonds de garantie sécurise les capitaux suivants:

- capitaux de couverture actuels des assurances de capitalisation;

- les parts excédentaires créditées;

- provisions pour prestations d'assurance en suspens.

En d'autres termes, la valeur actuelle de chaque assurance-vie avec l'intérêt épargné est à tout moment garantie. Ces dispositions font donc de l'assurance-vie un instrument de placement des plus sûrs. Ci-dessous une brève comparaison des divers placements les plus importants:

\begin{tabular}{|l|l|l|l|}
\hline & Sécurité & Rendement avant impôts & Disponibilité \\
\hline Assurance-vie & Fond de garantie & $3,70 \% *$ & Période fixe \\
\hline Compte épargne & $\begin{array}{l}\text { Garantie de l'Etat } \\
\text { actuelle jusqu'à Fr. } 30000\end{array}$ & $0,875 \%$ & A tout moment \\
\hline Emprunt d'Etat & Garantie d'Etat & $2,90 \%$ & Période fixe \\
\hline Obligations & Garantie par l'émetteur & Différent selon les émetteurs & Période fixe \\
\hline Obligations de caisse & Garantie par la banque & Selon la durée & Période fixe \\
\hline Or & Fluctuation du cours & Selon le cours & A tout moment \\
\hline
\end{tabular}

* lors d'un taux d'imposition max. de $30 \%$.

Un investisseur doit aujourd'hui plus qu'à n'importe quel moment se demander quels sont pour lui les principaux critères de choix. Par le passé, les produits n'étaient souvent comparés que par leurs différents taux de rendement. Mais que rapporte donc une promesse de rendement élevé si lors de la durée de validité l'émetteur devient insolvable? C'est avec plaisir et intérêt que nous vous conseillons dans ce domaine en vous indiquant les meilleures alternatives de placement.

\section{Talon réponse}

Prière d'envoyer ou de faxer au: 0319595010

Prénom / Nom

Adresse

NPA / Lieu

Date de naissance

Téléphone privé/cabinet

Atteignable le plus facilement (heure)

Adresse e-mail

Prière de me téléphoner pour un conseil personnalisé.

Je m’intéresse à:

\footnotetext{
Assurance vie

3 $3^{\mathrm{e}}$ pilier lié a

Planification financière

Caisse de pension LPP

Optimalisation fiscale

Responsabilité civile prof.
}

O
Roth Gygax \& Partner AG E Service de coordination
S E I C E s

Roth Gygax \& Partrasse 2 - 3073 Gümligen

Téléphone 0319595000 — Fax 0319595010

mail@fmhinsurance.ch wwww.fmhinsurance.ch 